\title{
Sobre la misteriosa invención de los psicotrópicos*
}

\author{
Philippe Pignarre \\ Traducción del francés al español de Luis Alfonso Paláu-Castaño \\ Universidad Nacional de Colombia, Medellín, Colombia \\ lapalau@gmail.com
}

\begin{abstract}
Tenemos acá una casi-paradoja. Alguna cosa, innegable, que no existía antes, resulta de la acción de contabilizar. $Y$ sin embargo esa cosa siempre ha sido verdad. En un sentido usted la crea, en otro sentido usted la descubre.
\end{abstract}

(William James)

Volvamos por un instante sobre la acusación lanzada contra la psiquiatría moderna: ella confundiría a los animales y a los humanos. Se podría responder que -incluso si se lo hace con un poco de mala fe, como se lo verá luego- si los psicoanalistas espantados tuviesen razón, es decir, que si una tal confusión resultase de un error, hace mucho tiempo que se hubiera detenido la búsqueda de poner a punto psicotrópicos eficaces probándolos primero en animales. Simplemente, porque esto no sería eficaz. Las investigaciones llevadas a cabo de esta manera serían estériles.

Hay pues, ciertamente, "alguna cosa" común en ratas, ratones, perros y seres humanos, más allá del soma, o a partir del soma. Después de todo, se puede tener confianza en la industria farmacéutica al menos en un punto: solo el resultado cuenta. Pero se está sobre una falsa pista si se cree que esa "cosa" está determinada por adelantado, que depende de una teoría de los animales, por ejemplo, de lo que tienen de común y de diferente con la especie humana. De hecho, esa "alguna cosa" que circula entre las ratas y los humanos no está dada por adelantado en las experiencias de los farmacólogos. Está en perpetua invención. Por lo demás, esta es la razón por la cual es tan difícil establecerla y caracterizarla. Se sabe que esa "cosa" es medible. Pero el error estaría en hacer

Cómo citar: Pignarre, P. (2020). Sobre la misteriosa invención de los psicotrópicos. Ciencias Sociales y Educación, 9(18), 287-300. https://doi.org/10.22395/csye.v9n18a15

Traduccción realizada por Luis Alfonso Paláu-Castaño del texto de Pignarre (2008).

Recibido: 10 de abril de 2020.

Aprobado: 21 de agosto de 2020. 
un resumen: decir "es el sufrimiento psíquico" y añadir inmediatamente "pero no es medible". Pensar la nueva biopsiquiatría con nuestras viejas categorías salidas de la psicopatología tradicional o del psicoanálisis, es hacer imposible todo análisis.

Es preciso pues comenzar a interesarse en lo que hacen los investigadores de la industria farmacéutica con las ratas y los ratones, aprender lo que ellos fabrican juntos con el fin de salir de las grandes generalidades bien pensantes. El problema aquí - y esta es una dificultad permanente en este género de debates - es estar arrinconados entre una "biopsiquiatría" que nunca dice la verdad sobre ella misma (lo más a menudo para garantizar el porvenir de sus financiamientos, pero también para colarse en las modas epistemológicas del momento y participar de la buena imagen de las neurociencias) y un psicoanálisis que toma por dinero contante la manera en que esta biopsiquiatría habla de sí misma. Los psicoanalistas nunca han tratado de saber cómo ocurre todo esto, concretamente, en los laboratorios de investigación; se contentan con retomar por su cuenta lo que los biopsiquiatras cuentan. Esto se volvió un juego de papeles. Los psicoanalistas acusan a los biopsiquiatras de ser reduccionistas (ellos reducirían los trastornos mentales a una diferencia biológica). Los biopsiquiatras están muy orgullosos de exhibir su reduccionismo que es, según ellos, la vía del progreso y la garantía de nuevas subvenciones.

¿Cómo hacer estallar este consenso facticio? Una manera es instalarse en toda la mitad y mostrar — como quiero hacerlo- que nada ocurre como se lo pretende o como se acepta creerlo. ¿Describe bien el "reduccionismo" lo que hacen y lo que saben los biopsiquiatras? ¿No son más bien "proliferadores" que multiplican los nuevos entes? Henos pues regresados a la cuestión de la ciencia o, al menos, de una ciencia particular: la farmacología o farmacodinamia, en tanto que subdominio de la biología.

A partir de 1952, fecha de introducción al mercado del primero de los psicotrópicos modernos, estos instalan lo que creo necesario llamar una "máquina" en medio de la "escena" psy, algo que las lobotomías eran incapaces de hacer, ni siquiera cuando Walter Freeman adoptó un comportamiento maquínico precursor a este respecto: lobotomizaba decenas de personas por día en una verdadera locura paranoica que solo pudo detener la llegada de la clorpromazina.

Si la clorpromazina hubiera sido un medicamento milagro en la esquizofrenia, el movimiento se habría detenido ahí. Lo mismo aplica para la imipramina en la depresión. No habría habido sucesores. La industria farmacéutica tuvo la suerte de encontrar un medicamento que, como todos los que van a seguir, marchaba 
bastante mal y era muy poco específico. Se necesitaba siempre sintetizar nuevos, haciendo "más o menos" la misma cosa. El "más o menos" está en el origen de la diversificación de las indicaciones.

Para hacer esto no se requería las neurociencias, sino solamente farmacólogos que trabajaban con perros, ratas, ratones, órganos y células para seleccionar las moléculas, buscando identificar las que tienen "más o menos" el mismo efecto que sus predecesores sobre los animales normales. No es de neurociencias de lo que se trata acá (sería ya demasiado noble como designación), sino que habría que hablar más precisamente de "pequeña biología" sin ninguna pretensión de fundar lo que merecería el nombre de "psiquiatría biológica".

Los psicotrópicos han reorganizado primero la psiquiatría pesada, heredera de la psiquiatría asilar, la que se ocupa de los pacientes psicóticos o esquizofrénicos. ¿Cómo trabaja la "máquina"? Ella es muy modesta al comienzo. No tiene pretensiones. Se presenta solamente como una herramienta suplementaria al servicio de los profesionales. Quiere solamente ayudar... No tiene ningún deseo de subirse a la escena a mostrar sus músculos. No sabe reír porque no se burla de nadie. Es respetuosa y, cada vez que un intermediario habla en su nombre, precisa que la acción de los psicotrópicos debe ser completada por una "psicoterapia". No es asunto de pelearse con nadie. Mientras que los lacanianos ocupan la escena pública, cantan y danzan, ella trabaja en el subsuelo sin que nadie se interese demasiado en sus maneras de hacer, en sus ambiciones. La máquina avanza lentamente, pero ella es menesterosa y nada parece poder detenerla.

La nueva "máquina" es bastante extraña; no posee una entrada y una salida como cualquier máquina común y corriente. Posee solamente dos salidas. Pero lo que sale por un lado es absolutamente indispensable a lo que sale por el otro. Sin esta doble salida, la máquina se detendría, sus fabricaciones se debilitarían, perecerían. Lo que sale por un lado nutre lo que sale del otro.

La primera salida deja pasar los productos de la pequeña biología. Se trata del conjunto de las técnicas que van a permitir perfeccionar nuevos psicotrópicos, de hecho: "sucesores" de los que ya están en el mercado. Las características de la máquina explican por qué los nuevos psicotrópicos nunca son muy diferentes de los antiguos. La salida técnica de la máquina tiene ante todo rodamiento un cierto número de pruebas. Fueron primero test comportamentales sobre animales, esencialmente sobre ratas, ratones o perros. Estos ensayos datan de mediados de los años cincuenta, y comienzan por ser observacionales. Por ejemplo, ¿cuánto tiempo permanece un ratón agarrado a un tronco que gira, antes de caer, después de la absorción o no del candidato a medicamento? También hay test de reversión: ¿cómo un candidato a medicamento invierte los efectos de otra sustancia en el perro (suprimir el vómito inducido por la apomorfina, 
por ejemplo, para encontrar neurolépticos de los que se sabe que siempre son antieméticos $\left.{ }^{1}\right)$ ? Más tarde, se inventan experimentos celulares: ¿cómo un candidato a medicamento actúa sobre los receptores neuronales o sobre sustancias que circulan en el cerebro (como la dopamina)?.

Estos test de comportamientos o bioquímicos no son inventados in abstracto, por ejemplo, para tratar de imaginar lo que podría ser una rata esquizofrénica o un ratón depresivo. Son siempre inventados con referencia a lo que hacen los psicotrópicos ya en el mercado, y cuyo efecto psíquico siempre ha sido descubierto de manera fortuita. Si un psicotrópico ya utilizado en clínica humana induce tal o cual efecto comportamental o bioquímico en el animal, se va a buscar todas las moléculas que producen más o menos el mismo efecto. El "más o menos" es aquí muy importante, pues, para un equipo de investigadores de un laboratorio farmacéutico, perfeccionar un medicamento precisamente "un poco" diferente de los precedentes, es muy prometedor desde un punto de vista comercial.

Desplazándose así, los psicotrópicos se han diversificado progresivamente y han invadido todo el campo de los trastornos mentales. Muy rápido, se ha buscado moléculas que sean menos potentes que sus predecesoras, pues al poner a punto versiones light de los hipnóticos, de los neurolépticos y de los antidepresores existentes, se han podido extender las indicaciones y transformar así a los médicos generales en prescriptores de psicotrópicos. Se ha vuelto el caso desde comienzos de los años ochenta y la ventaja es doble: menos poderosos los psicotrópicos tienen menos efectos secundarios, y se puede justificar su prescripción a personas menos gravemente afectadas.

Pero este es solo la mitad del asunto. Al lado de esta pequeña biología suma de las herramientas técnicas para inventar medicamentos exitosos-, la máquina produce, simétricamente, una "pequeña psicología" que está camino de invadir y de volver a desplegar, de una nueva forma, el conjunto del campo psy. Y es sobre todo a esta pequeña psicología con la que van a tropezar de ahora en adelante los psicoanalistas; la van a encontrar en permanente evolución, pero siempre igualmente tonta. Les será difícil comprender cómo, a pesar de su indigencia teórica, ella puede triunfar sobre la antigua psicopatología. Tampoco comprenderán por qué las batallas ideológicas no impedirán su triunfo.

Se puede seguir los efectos de organización y de reorganización inducidos por la pequeña psicología: estudios comparativos que se han vuelto obligatorios (a partir de 1962, por los poderes públicos); creación de herramientas de diagnóstico y escalas comportamentales para poder comandar tales estudios; perfeccionamiento y refinamiento de esos útiles al hilo del tiempo y de la llega-

Es así como la metoclopramida (el Primperán) es utilizado como antiemético mientras que inaugura una nueva familia de neurolépticos, las benzamidas sustituidas, cuyo último avatar es el Solian. 
da de nuevos psicotrópicos. Se abandona progresivamente la división psicosis/ neurosis, inútil para juzgar la eficacia de los medicamentos y que podría incluso frenar su utilización, para tratar síntomas como la ansiedad que se reencuentra en patologías muy diferentes más o menos graves. Nuevas entidades clínicas dominan la escena. La noción de depresión juega un papel clave en este proceso de reorganización. Incluso los psicoterapeutas pueden ser puestos al servicio de la máquina cuando le abren nuevos territorios: trastornos obsesivos compulsivos (los TOC), síndrome postraumático. Muy rápido, la farmacología bombardea estos nuevos campos con moléculas, los reformula y se apodera de ellos. iQué extraordinario camino recorrido desde los tiempos de la lobotomía! En esa época, el principal criterio utilizado para medir la eficacia de este tratamiento era el número de pacientes capaces de abandonar el hospital y de regresar a vivir en su familia. Actualmente, se considera que esto era poco. Es solamente con los psicotrópicos cuando se van a multiplicar las escalas, los test, toda esa extraordinaria batería que permite medir el efecto de los medicamentos, de compararlos entre ellos. Comienza una verdadera revolución.

Si para los psicoanalistas hay un continuo entre lo normal y lo patológico, para la nueva psiquiatría el continuo está entre las perturbaciones graves y los trastornos leves (las psicosis y las neurosis). La acción misma de los medicamentos psicotrópicos permite pensar esta continuidad; pueden ser utilizados de manera "transnosológica".

Las psicoterapias son, finalmente, redefinidas y reorganizadas; serán comportamentalistas. Se adaptan así a las nociones, a las exigencias y a los protocolos que convienen a los medicamentos psicotrópicos. Utilizan de acá en adelante las mismas definiciones de los trastornos psicológicos, los mismos criterios de mejoría, las mismas escalas y las mismas herramientas estadísticas que aquellos que han sido concebidos para probar la eficacia de los psicotrópicos. Pueden, pues, sin problema, ser coprescritos y sus efectos conmensurados. Por lo demás, con bastante frecuencia, tendrán por principal objetivo enseñarles a los pacientes a tomar bien sus tratamientos medicamentosos...

La máquina inventa pues, por un lado, los útiles que permiten medir los efectos de lo que ella produce por el otro. Soltaron la palabra: de ahora en adelante se puede "medir".

La doble producción de la máquina farmacéutica crea la posibilidad de medir. La máquina para inventar psicotrópicos permite crear "tensores" que van a admitir la medida. Esos "tensores" son las producciones de la máquina, por ejemplo, entre psicotrópicos energizantes (llamados antidepresivos) y la 
depresión redefinida por el DSM; o también entre los tranquilizantes mayores (que son los neurolépticos) y la esquizofrenia redefinida por el DSM.

Dos errores simétricos deben ser evitados aquí. El primero es el más corriente: creer que el "sufrimiento psíquico" es medido. Se mide lo que ocurre entre los nuevos tensores, es decir, algo sin precedente que no preexistía a la doble invención de los psicotrópicos y de la pequeña psicología. El segundo error sería creer que lo que se mide no tiene nada que ver con lo subjetivo. El error sería creer que medir implica "un progreso en la frialdad, en la distancia y en la objetividad...". Se puede retomar acá la fórmula que Bruno Latour y Vincent Antonin Lépinay (2008) aplican a la economía: "Nada en la economía es objetivo, todo es subjetivo o, más bien, intersubjetivo, y esta es precisamente la razón por la cual se la puede hacer cuantificable y científica" (p. 17). Propongo llevar aún más lejos esta comparación con lo que estos dos autores dicen de la economía:

Al volverse la medida "más simple", el "estado social" se ha vuelto reflexivamente más fácil de descubrir. Conviene pues distinguir bien dos tipos de medida: la que atraparía el estado real (que se podría llamar la medida mesurada) y la que formatea el mundo social (y que se podría denominar la medida mesurante) [...]. Introduzcamos la palabra valorímetro para calificar todos los dispositivos que permitan hacer visibles y legibles los juicios de valor que forman el fondo de lo que Tarde va a llamar economía. Se imagina sin dificultad su interés para la época actual que ve multiplicarse las formas del audimat ${ }^{2}$, de los sondeos [...] tan preciosos para "entregar el estado social más sujeto al número y a la medida". Se trata de ir a "buscar en cada tipo de práctica los tensores que le son particulares". (Latour y Lépinay, 2008, pp. 29-30)

Los psicotrópicos solo fueron un acontecimiento, en el sentido fuerte del término, porque se hicieron acompañar de la invención de una pequeña psicología (evolutiva como los propios psicotrópicos). Y esta doble invención permite medir, crear "mentalómetros" (atrevámonos con esta palabra). Estamos plenamente en la "medida mesurante", mientras que los biopsiquiatras, agarrados en las palabras por los psicoanalistas, quieren hacernos creer que ellos están en la "medida mesurada". Se podría llevar aún más lejos la analogía con la economía. Allá donde el francés solo dispone de una sola palabra, "economía", la lengua inglesa propone dos: "economics" y "economy", es decir, "la economía-cosa" y "la economía-disciplina". Ahora bien, claramente la segunda es la que performa ${ }^{3}$

2 Etimología de 1981, nombre patentado, de audí(metro) y auto(mát)ico; audímetro conectado a la red telefónica que permite medir la audiencia de las cadenas de televisión (Petit Robert, electrónico). Nota del traductor.

3 Siguiendo la indicación de Austin de que un performativo [el acto performático] es el que describe una determinada acción del locutor al mismo tiempo que su enunciación equivale al cumplimiento de ella, Pignarre afirma que la economy ejecuta la economics, o que la pequeña psicología crea el estado de cosas psicológico. Nota del traductor. 
y formatea la primera ${ }^{4}$. Será menester igualmente distinguir la "psicología-disciplina" y la "psicología-cosa". La primera no es el reflejo de la segunda, como lo podría creer un empirismo ingenuo, sino que ella la performa y la formatea.

No hay deus ex machina, solamente pequeñas manos como esos grandes patronos de la psiquiatría (los "líderes de opinión"), que ponen su notoriedad al servicio de la máquina. El terreno está de acá en adelante minado por todas partes. Las pobres pequeñas máquinas freudiana y lacaniana están en vía de desaparición, o de convertirse en una simple referencia cultural que ya casi no encuentra ningún eco en los dispositivos de cura; ellas han sido marginalizadas y, lo que es quizás más difícil de comprender por parte de los psicoanalistas, sin ningún debate. No hay ninguna seguridad de que las psicoterapias que no juegan ningún papel en la máquina (por ejemplo, desbrozando nuevos territorios de cuidados psicológicos, o viniendo a "completar" la acción de los medicamentos) continúen existiendo durante mucho tiempo, a no ser en las márgenes del sistema, es decir, sin ser pagadas por la seguridad social, por ejemplo.

La noción de "máquina" que me parece indispensable para tratar de comprender el modo de invención de los psicotrópicos, ha suscitado la ira de los psicoanalistas humanistas. iCuántas veces Élisabeth Roudinesco me ha reprochado que hable de máquinas, creyendo que yo hacía su elogio! Yo tomé prestada esta noción de Gilles Deleuze y Félix Guattari, recordemos el comienzo de El anti-edipo: estamos en una "fábrica", no en un "teatro". En este sentido, escriben Guattari y Rolnik:

Es necesario distinguir las máquinas de la mecánica. La mecánica está relativamente cerrada sobre sí misma; sólo mantiene con el exterior relaciones relativamente codificadas. Las máquinas consideradas en sus evoluciones históricas, constituyen por el contrario un filum comparable al de las especies vivientes. Se engendran las unas a las otras, se seleccionan, se eliminan, haciendo aparecer nuevas líneas de potencialidades. Las máquinas en el sentido amplio (es decir no solamente las máquinas técnicas, sino también las máquinas teóricas, sociales, estéticas, etc.) nunca funcionan aisladamente sino por agregación o por disposición. Una máquina técnica, por ejemplo, en una fábrica, está en interacción con una máquina social, una máquina de formación, una máquina de investigación, una máquina comercial, etc. (pp. 459-460)

La máquina produce, pues, nuevas entidades moleculares de las cuales ella encuentra medianamente las indicaciones posibles. A la "pequeña biología" le corresponde así (como lo hemos visto) una "pequeña psicología" que se inventa

"La disciplina económica inventada en el siglo XVIII, no descubre un continente, lo fabrica por completo o, más bien, lo organiza, lo conquista, lo coloniza" (Latour y Lepinay, 2008, p. 27). Ver también, por ejemplo, Michel Callon (1998). 
simultáneamente con los medicamentos. Es lo que arrebatan, más o menos bien, las diferentes moléculas. El DSM juega el papel de guardar la frontera entre lo normal y lo patológico. Así, se reserva el derecho de hacer siempre nuevas incursiones para anexar dominios que eran considerados antes como normales.

Entre las moléculas y sus indicaciones, es siempre un poco la alianza del ciego y del paralítico ${ }^{5}$. Si se trata de separar las moléculas de las indicaciones, se tiene bastante dificultad para caracterizarlas; son pharmakon, a la vez veneno y remedio. Solamente se las puede aproximar viendo lo que hacen en algunas circunstancias. Si usted separa la mayor parte de las indicaciones de las moléculas que le están destinadas, ellas pierden igualmente mucho de su evidencia. Fue lo que ocurrió con el reporte del Inserm sobre los trastornos de conducta en el niño y en el adolescente que - queriendo instituir independientemente su pequeña psicología - se fracasó en el escepticismo de los clínicos. En desquite, no nos engañemos; juntos, el ciego y el paralítico, tienen una cierta eficacia y mantienen la ruta.

Como en todos los casos en los que se tiene que ver con una máquina, la cuestión de la escogencia no solamente no se plantea, sino que parece sin interés, desplazada, casi idiota en tanto todo parece ser evidente.

Si se mira de más cerca, nos damos cuenta de una diferencia considerable entre este laboratorio de perfeccionamiento de nuevos medicamentos y el laboratorio del químico, por ejemplo. Quizás yo me equivoqué en el pasado al confundirlos en demasía. Funciona incluso a la inversa del laboratorio científico clásico; mientras que este último trabaja sobre fenómenos puros (la caída de los cuerpos, pero sin el frotamiento), nuestro curioso laboratorio se esfuerza en "despurificar" los fenómenos que quiere observar. Se interesa en la manera en que su producto migra en el mundo, es decir, se aproxima por etapas del fenómeno sumergido en un entorno normal, lo que se llamará la "naturaleza" o la "sociedad", y este producto va a ser modificado por todos los actores sucesivos que preparan su entrada en el mundo. Así, se va a alejar el lead original

\footnotetext{
Es lo que, en El parásito, Michel Serres (año) (por favor citar adecuadamente El parásito y agregar a la lista de referencias) ha contado como apólogo: "Un paralítico se arrastraba sobre los codos y las rodillas. [...] A cuatro pasos de una suculenta comida uno se puede morir de hambre, cómo Tántalo, si no se puede desplazar. Se moría de miseria y se pudría en un rincón oscuro. Un buen día, vio a un ciego que tropezaba con mil obstáculos y que arriesgaba con desnucarse. Puede morir cayendo a un pozo si el brocal es bajo y se le parece a una escala, y si los brazos tendidos sólo tocan el viento. El inmóvil lo llama y le ofrece un contrato. El ciego lo llevará y el estropeado lo guiará. Entre los dos hacen un normal". El tullido propone al ciego el pacto parasitario. Anunciará el obstáculo, vigilará, propondrá la dirección, clarificará, iluminará (información de escala microscópica). El ciego es la fuerza, el transporte, potencia calculable en calorías (energía de escala ordinaria)." (pp. xx-xx). Nota del traductor. (Como esta es una nota del traductor, se sugiere citar y referenciar la fuente, pues esta nota no hacía parte del original francés. Gracias.)
} 
añadiéndole o recortando algunos átomos, luego se modifica la farmacodinamia y la biodisponibilidad de la molécula seleccionada para obtener un medicamento que se tome una vez al día, etc.

Para hacer la distinción entre este dispositivo y el laboratorio precedente, Isabelle Stengers (2006) propuso llamarlo "laboratorio técnico moderno". Algunos sociólogos de las ciencias, como Michel Callon (1998), han escogido una fórmula que evita la palabra "laboratorio" y hablan de acá en adelante de "plataforma experimental" para insistir en el hecho de que se trata de un lugar de pasaje entre el interior del laboratorio y su exterior, a diferencia de un laboratorio puramente científico.

La noción "plataforma experimental" permite pensar la invención técnica de manera mucho más concreta de lo que permite la oposición clásica entre "ciencia fundamental" y "ciencia aplicada". Para hacer funcionar una "plataforma experimental", los investigadores inventan procedimientos dignos de un laboratorio científico, pero son hibridados de forma permanente. La separación con la naturaleza no está instaurada de una vez por todas y defendida de toda contaminación. La contaminación hace su entrada progresiva en el laboratorio para que se pueda mirar si el fenómeno estudiado "se mantiene siempre".

En el caso de los medicamentos, la "plataforma experimental" está constituida, entre otras cosas, por los ensayos clínicos de la fase I a la fase III (y todas las operaciones anexas, de trasfondo, como el perfeccionamiento de las sucesivas versiones del DSM, que son llevadas para tener en cuenta resultados obtenidos y ayudar a su integración en los estudios por venir). Se distinguen a menudo estas tres fases por sus diferentes objetivos; pero es igualmente importante observar que, en el curso de cada fase, el número de pacientes movilizado aumenta, hasta alcanzar muchos miles ${ }^{6} 5$. Se experimenta así la capacidad del "candidato a medicamento" para hacer su entrada en sociedad. Cantidad de cuestiones ajenas a la ciencia deben ser resueltas para que esta plataforma técnica funcione (la cuestión de las patentes, por ejemplo). El candidato debe reclutar nuevos aliados (clínicos, pacientes, autoridades de salud). Puede también modificarse para integrar nuevas obligaciones.

Pero el estudio de este laboratorio técnico nos enseña una segunda cosa, absolutamente decisiva. Contrariamente al modo como ella se presenta, la biopsiquiatría no funciona de ninguna manera poniendo en relación un trastorno mental, síntomas y una característica biológica o genética. Incluso ella

Después de los estudios animales, se pasa a los ensayos en el hombre: estudio de la farmacocinética en algunos individuos voluntarios sanos (fase I); evaluación de la eficacia terapéutica abierta a un pequeño grupo de enfermos hospitalizados (fase II); doble ignorancia en el momento de grandes estudios comparativos multicéntricos (fase III); finalmente, los estudios de fase IV que tienen lugar en un medio abierto que permiten afinar los datos concernientes a la tolerancia del producto a largo plazo (Dagognet, 2002). Nota del traductor. 
está totalmente organizada en torno a esta imposibilidad, y es allá donde se puede comprender su dinámica. Lo que la psiquiatría biológica pone en relación son sustancias químicas y síntomas, lo que va a entrañar (en el curso de esta operación) su reagrupamiento o, al contrario, su aislamiento. En este sentido, la biopsiquiatría inventa algo totalmente nuevo, y si abandona el tesoro de la vieja psiquiatría clásica, no es como consecuencia de un proyecto ideológico, sino porque aquel no le sirve para nada.

Los psicoanalistas han tratado de ampliar el campo de aplicación del psicoanálisis por vías teóricas. ¿Pero es esto suficiente para pasar de la psiquiatría de las ciudades a la psiquiatría de los campos, de la neurosis a la psicosis, del psicoanálisis a las psicoterapias? Es algo dudoso. Se podría comprender la prudencia de Lacan sobre estas dos cuestiones. La definición de la psicosis, en torno a la noción de forclusión del Nombre-del-Padre, refuerza la dificultad de un pasaje entre neurosis y psicosis, lo que lleva a Jean-Claude Maleval (2000) a reconocer: "Se comprende que Lacan se haya mostrado tan pesimista como Freud con respecto a la cura de los psicóticos por parte del psicoanálisis" (p. 55).

Escribe además:

En 1959, conducir el análisis de un psicótico a la manera del de un neurótico le parecía a Lacan "tan estúpido como fatigarse con el remo cuando el navío está ya en la arena"; porque estaba excluido que se pudiera analizar la forclusión. (Maleval, 2000, p. 133)

Añade que después de esa fecha, "su investigación sobre la psicosis no toma ya nunca más la forma de una elaboración tendiente a una formalización global" (Maleval, 2000, p. 111).

Esto confirma lo que sabe quien haya leído el seminario sobre las psicosis, en el cual Lacan no cesa de multiplicar las barandillas (garde-fous). Explica que no se tratará finalmente de hablar del "tratamiento de las psicosis", como lo había podido anunciar un poco imprudentemente al final del año precedente, y que no tratará de la esquizofrenia sino solamente de la paranoia.

El psicoanálisis no es, pues, el objeto de una gigantesca empresa ideológica de destrucción, bien organizada y teóricamente concertada. Si fuera el caso, habría oportunidades infinitamente mejores de salir de él. Él es mucho más claramente víctima de un progresivo desinterés, pues lo que importa pasa en otra parte, viene de otra parte. Y es solo en los márgenes, con frecuencia después de reacciones imperiosas de psicoanalistas, que el debate se inflama y toma la forma de una guerra de ideas. 
En un combate entre una "teoría" que reclama nuevas aplicaciones (psicoterapias, encargarse de psicóticos) y una "plataforma experimental" que produce estos objetos híbridos que son los medicamentos, la teoría no tiene ninguna oportunidad. ¿En qué se convierten, entonces, las viejas preguntas tradicionales sobre el "sufrimiento psíquico", sobre la imposibilidad de medirlo, sobre el carácter único de cada paciente? ¿Por qué la tentativa de los psicoanalistas de poner el debate en este terreno solo ha dado lugar es a trivialidades? Podría claramente haber aquí una "dimensión" de los psicotrópicos y de lo que se inventa simultáneamente con ellos, que los psicoanalistas no han sido capaces de captar y que los excluye progresivamente cada vez más de lo que está camino de pasar en psiquiatría, esto es, los pone de alguna manera por fuera del juego.

Un medicamento que da satisfacción a los pacientes y a los terapeutas en el curso de los ensayos clínicos "cuelga" algo que es del orden de lo subjetivo, pero no se confunde con lo que se entiende por "sufrimiento psíquico". Y es la acción del medicamento mismo el que va a permitir asediarlo mejor, cuantificarlo. En biopsiquiatría, no hay diagnóstico posible independientemente de los medicamentos. En este sentido, la biopsiquiatría está organizada en torno a una "carencia", la del diagnóstico independiente, que hace referencia a una variación biológica o genética. Ella existe según modalidades estrictamente opuestas a la manera como se presenta públicamente. Es interesante constatar que todas las nuevas herramientas técnicas, como la farmacogenómica, no han hecho sino reproducir, de forma ampliada, esta situación.

Y, sin embargo, hay claramente en esta operación "algo" que circula de un ensayo clínico al otro, que encontrará su reflejo aproximativo en las herramientas como el DSM, pero que no se limita allí. En efecto, ese "algo" circula también entre los animales de laboratorio y los seres humanos. Hemos aprendido a reconocer comportamientos animales (o de aquí en adelante: variaciones celulares) que hacen que ese "algo" que los atraviesa permita predecir un efecto psicotrópico en los seres humanos. Como los psicoanalistas, los farmacólogos no quieren oír hablar de ratones esquizofrénicos. Ellos no habrían inventado, entonces, nada importante. No es así como trabajan, sino descubriendo efectos predictivos en animales "normales". Los candidatos a psicotrópicos son señalados porque tienen, en animales, "más o menos" los mismos efectos que las moléculas ya existentes. Es así como se ha inventado algo radicalmente nuevo que, sin duda, merece un nuevo nombre; ya no "psiquismo", sino quizás "cuerpo mental".

Esta concepción tiene la ventaja de poner fin a un debate poco fructuoso sobre el hecho de saber si se puede o no "medir el sufrimiento psíquico". Es una cosa bien distinta la que los farmacólogos y los biopsiquiatras han aprendido a hacer circular y se esfuerzan por medir: un conjunto de "rasgos" que han aprendido 
a extraer de manera totalmente original y que no podrían, de ninguna manera, ser asimilados a lo que existía antes en psiquiatría.

El punto común de todas las prácticas que se interesan en lo que hemos llamado, de manera simplificada, el "psiquismo humano" hay que remitirlo a una técnica y no a un saber objetivo científicamente fundamentado. Interesándose en el proyecto de Tobie Nathan, Isabelle Stengers (2006) escribe:

La propuesta de Tobie Nathan según la cual los curanderos deberían ser reconocidos como técnicos - los que saben los procedimientos técnicos cuya eficacia puede estar asociada a lo que llamamos curación— aclara la devastación ecológica asociada a la definición de las técnicas llamadas modernas. Si una técnica al fin digna de ese nombre debe derivar de un saber objetivo válido, las técnicas del curandero que entran en relación con los djinnes ${ }^{7}$ o con divinidades, deberán ser comprendidas a partir de lo que ponen en operación sin que ellas lo sepan (efecto placebo, eficacia simbólica, poder performativo del lenguaje, sugestión, transferencia, etc.). En desquite, si la práctica técnica es primera, si su eficacia está liberada de su sujetamiento a lo que la explicaría, la definición por "fin moderna" de lo que hemos llamado perturbación psíquica se pone a sonar terriblemente vacía, porque sus "causas" psicoanalíticas, psiquiátricas, neurofisiológicas, están hechas ante todo para explicarla mucho más que para permitir pensar, percibir y operar a un médico. (pp. 199-200)

Esta tarea debe permitirnos escapar del dualismo que encontramos habitualmente en el discurso de los psicoanalistas cuando ellos hablan de los medicamentos al oponer el cuerpo y la subjetividad. Esa cosa que se trasfiere de los animales a los humanos (en los laboratorios de investigación de la industria farmacéutica), luego de los humanos a los humanos (en los estudios clínicos) es una "materialidad abstracta". No se trata aquí de escribir la fórmula química del medicamento que nos habla de otra cosa, sino la del conjunto de las reacciones microscópicas y en cascada que provoca una molécula, las puntadas de basta que hacen que una molécula tenga efectos, haga algo que pueda ser transportado... y medido.

Esta dimensión de los medicamentos puede estar relacionada con el interés que ellos han suscitado entre los pacientes. No es porque los pacientes sean disciplinados que han acogido bien los psicotrópicos, es porque esos psicotrópicos tienen una eficacia que da a los pacientes la libertad de volverse a fabricar. Esto evidentemente no sería posible si la biopsiquiatría pusiese en relación una

Un djinn es un genio de la mitología árabe, un espíritu del aire. El más famoso de todos es el de la lámpara de Aladino, aunque si lees Las mil y una noches encontrarás una buena colección de ellos. Por lo general se les muestra dotados del poder de conceder deseos, suelen aparecer como espíritus benévolos, en contraposición a los ifrit, genios del fuego, malignos. Nota del traductor. 
causa biológica y un medicamento, como lo creen los psicoanalistas que toman en serio las mezcolanzas teóricas de los biopsiquiatras.

La existencia de este "laboratorio técnico" (o "plataforma experimental") donde se inventan los medicamentos ponen a los psicoanalistas en situación difícil. ¿Cómo un médico analista puede, al mismo tiempo, prescribir psicotrópicos y continuar proclamando que "no se puede medir el sufrimiento psíquico" ${ }^{8}$ ? Los médicos analistas nunca han tomado claramente posición sobre este laboratorio técnico:

- ¿Aceptáis la existencia de esta plataforma como lugar de paso de los candidatos a medicamentos al estatuto de medicamento?

- En caso contrario, ¿qué procedimiento proponéis antes de decidir si un psicotrópico puede ser introducido en el mercado?

Pongo a propósito a los médicos analistas en una posición difícil. Pues si aceptan el papel de esta plataforma experimental, no se ve con qué argumentos ellos pueden oponerse (una vez salidos los medicamentos del laboratorio) a su prescripción según las mismas reglas, las mismas indicaciones, etc.; excepto que se las prescriba mal, es decir, a ciegas io en función de criterios que no eran utilizables para probarlos bien! En su estudio del funcionamiento de los equipos psiquiátricos en un hospital de Buenos Aires, el sociólogo de las ciencias Andrew Lakoff (2008) mostró que los médicos psicoanalistas prescriben más neurolépticos que sus colegas biopsiquiatras desde que sospechan una estructura psicótica, es decir, desde que están en presencia de un delirio y de alucinaciones, lo que engloba la esquizofrenia, la melancolía, la psicosis maníacodepresiva, bajo el paradigma de la paranoia (insuperables los casos Schreber y Aimée). En cuanto a los biopsiquiatras, no prescriben un neuroléptico a los pacientes que sufren de lo que ellos llaman un "trastorno bipolar" (cuidándose de no irlo a llamar psicosis), sino que ensayan primero un estabilizador del humor. Procediendo así —incluso si dudan y hacen del estabilizador una especie de "ensayo terapéutico" - hacen una escogencia favorable a los pacientes. Pues se sabe que los efectos secundarios de los neurolépticos hacen que los pacientes se parezcan a esquizofrénicos y que iellos terminan siempre por darle la razón a los que los prescriben!

Se podrían aún multiplicar las citas. Digamos lo que escribe, por ejemplo, la psicoanalista Patricia Johansson-Rosen (2007): "A veces es preferible no prescribir medicamentos [en caso de duda sobre la estructura, psicótica o neurótica]

Esta frase ya célebre, fue pronunciada por P. Douste-Blazy (2005), entonces ministro de Salud. 
sino... volver a ver rápidamente al paciente. O prescribir de entrada una pequeña dosis de neuroléptico (pp. 69-77).

\section{Referencias}

Callon, M. (1998). Laws of the Markets. Wiley-Blackwell.

Dagognet (2002). El cerebro ciudadela (L. Paláu, trad.). Facultad de Ciencias Humanas y Económicas, Universidad Nacional de Colombia.

Douste-Blazy, P. (2005, 4 de febrero). Discours Psychiatrie et Santé mentale Projet de plan 2005-2008 [conferencia de prensa]. https://url2.cl/YPlmL

Guattari, F. y Rolnik, S. (2007). Micropolitiques. Les Empêcheurs de Penser en Rond.

Johansson-Rosen, P. (2007, 19 de mayo). El DSM-IV, el medicamento y el psicoanálisis. Mental.

Lakoff, A. (2008). La Raison pharceutique. Les Empêcheurs de Penser en Rond.

Latour, B. y Lépinav, V. (2008). L'économie, science des intérêts passionnés. La Découverte.

Maleval, J. (2000). La Forclusion du Nom-du-Père. Le concept et sa clinique. Seuil.

Pignarre, P. (2008). Sur la mystérieuse invention des psychotropes. En Philippe Pignarre, La cigale lacanienne et la fourmi pharmaceutique (pp. 40-60). Epel.

Stengers, I. (2006). La Vierge et le neutrino. Les scientifiques dans la tourmente. Les Empêcheurs de Penser en Rond. 